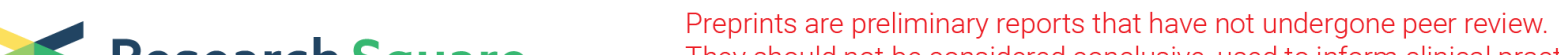 Research Square They should not be considered conclusive, used to inform clinical practice, or referenced by the media as validated information.
}

\section{Mel Aggravates Pyroptosis Induced by Temozolomide Through Inhibiting NRF2-are Pathway in Glioblastoma}

Hao Pan

East Region Military Command General Hospital Handong wang ( $\nabla$ njhdwang@hotmail.com ) Qiang Wang

East Region Military Command General Hospital

Wenhao Niu

East Region Military Command General Hospital

Qi Wu

East Region Military Command General Hospital

Yue Jia

East Region Military Command General Hospital

Kangjian Sun

East Region Military Command General Hospital

Longbang Chen

East Region Military Command General Hospital

Research

Keywords: Melatonin, Pyroptosis, Nrf2, Glioblastoma

Posted Date: June 17th, 2020

DOl: https://doi.org/10.21203/rs.3.rs-35576/v1

License: (c) (i) This work is licensed under a Creative Commons Attribution 4.0 International License.

Read Full License 


\section{Abstract}

Background:Glioblastoma(GBM) is a common malignant tumor of the brain. It has been verified that melatonin(MEL) can inhibit glioma proliferation. But its mechanism has not been fully elucidated. We intend to examine the mechanism of MEL on glioma cells from the perspective of pyroptosis and Nrf2.

Methods:Expression of MEL receptor in glioma was detected by western blot. GBM cell viability treated with temozolomide(TMZ) plus MEL was detected by CCK-8. Pyroptosis rate was determinate by flow cytometric analysis. Western blot was used to detect the Nrf2 and pyroptosis related protein level after MEL treatment. Orthotopic tumor growth inhibition study was performed to further investigate the tumor inhibition effect of TMZ plus MEL.

Results:We first confirmed MEL receptor was abundant in glioma tissue and cell lines. After combined treatment of TMZ and MEL, cell viability decreased significantly as compared to those of TMZ treatment alone. Also, the ratio of pyroptosis and ROS level increased, followed by elevated expression of pyroptosis related protein. Furthermore, MEL can induce a diminution of Nrf2 expression in glioma in dose- and time-dependent manner. TMZ can increase Nrf2-ARE pathway expression, which also can be deprived by MEL. Its inhibition of Nrf2 depends on dephosphorylation of IGF-1/AKT/mTOR pathway. More importantly, after overexpression of Nrf2 in glioma cells, the level of pyroptosis-related protein elevated by MEL decreased, suggesting that the effect of MEL on promoting pyroptosis is dependent on its inhibition of Nrf2. In vivo results further confirmed that MEL plus TMZ induced significantly decreased tumor size and increased pyroptosis rate, but had no significant effect on mouse body weight, ALT, AST.

Conclusion:MEL can inhibit the phosphorylation of IGF-1/AKT/mTOR pathway at millimol level, which further reduces the expression of $\mathrm{Nrf} 2$ and promotes pyroptosis of glioma cells. Considering the modest efficacy of TMZ chemotherapy, MEL can be considered as a potential chemotherapy sensitizer to improve the chemotherapy effect of glioma.

\section{Background}

Glioma accounts for $30-40 \%$ of all intracranial tumors. Glioblastoma(GBM), which is the most lethal primary brain tumor, is the most familiar and malignant glioma[1,2]. Treatment strategies, such as combination of aggressive resection, radiotherapy and temozolomide (TMZ) plus many new chemotherapeutics, are performed in treatment of malignant glioma. But the median survival time is still extremely poor[3]. Taking into account for the serious side effect of extensive tumor resection and high dose radiotherapy or chemotherapy, the chemosensitizer may be a new way to better survival.

Melatonin (N-acetyl-5-methoxytryptamine, MEL) is an indolamine secreted by the pineal gland that is intricately involved in the regulation of human chronobiological rhythms and endocrine function. Many reports in recent decades paid attention to antitumor effect of MEL in many kinds of tumors, including glioma. It has been noted that melatonin in $1 \mathrm{mM}$ significantly inhibited the migration and invasion of human U251 glioma cells under hypoxia[4]. Also MEL can reduce migration and invasion of U251 and 
T98G glioma cell with inhibition on matrix metalloproteinase 9 (MMP9) and MMP2 and diminution of intracellular free radical generation[5]. MEL alone also reduces the stem cell phenotype of glioblastomainitiating cells (GICs) and induces diminished proliferation and enhanced autophagy of GICs [6]. And combination of MEL and TMZ has a synergistic toxic effect on glioma cells, which is correlated with increased methylation level of ABCG2/BCRP promotor[7]. All these results indicate that MEL is a potential chemosensitizer in glioma treatment.

Here we reported a new mechanism under anti-glioma effect of MEL. Our results indicated that MEL inhibited the basal level and TMZ induced nuclear translocation of Nrf2, an important antioxidant transcription factor. With diminished Nrf2 level in glioma cells, elevated ROS level induced enhanced pyroptosis in vivo and in vitro after TMZ treatment.

\section{Methods}

\section{Cell Culture}

Human U251, A172, U118, T98 and U87 glioblastoma cells were obtained from Chinese Academy of Sciences Committee Type Culture Collection cell bank and cultured in DMEM (HyClone, IL, USA) with 10\% FBS (Wisent, Nanjing, China). Normal human astrocyte (HA) primary cultures were obtained from the Institute of Basic Medical Sciences (Beijing, People's Republic of China) and maintained in their patented medium.

\section{Patient samples}

Glioma patient specimens for analysis were collected from 2017 to 2019 in Department of Neurosurgery, Jinling Hospital, after informed written consent was obtained from each subject or each subject's guardian according to the institutional guidelines and the Declaration of Helsinki principles. The tissue was collected and stored in liquid nitrogen until the analysis.

\section{CCK-8 assay}

Cells in $100 \mu \mathrm{l}$ were seeded to 96 well culture plates at $2 \times 10^{3} /$ well. $24 \mathrm{~h}$ after seeding, TMZ or TMZ plus different concentration of MEL was added to wells and cultured. Cell survival rate was quantified using a Cell Counting Kit-8 (Dojindo, Japan) at different time according to the manufacturer's protocol. Briefly, $10 \mu \mathrm{l} \mathrm{CCK}-8$ was added and incubated at $37^{\circ} \mathrm{C}$ for $1 \mathrm{~h}$. Then the OD value was read at $450 \mathrm{~nm}$ using a Bio-Rad ELISA microplate reader (Bio-Rad Laboratories, USA). All measurements were performed in sextuplicate. Results were showed as mean \pm SD.

\section{Plasmid transfection}

Primers for human Nrf2 cDNA were: forward 5'-CCGCTCGAGATGATGGACTTGGAGCTGCC-3', reverse 5'GGGGTACCGTGTTTTTCTTAACATCTGGC-3'. Human Nrf2 cDNA was cloned into the cloning site of the vector pEGFP-N1 (GeneChem, Shanghai, China) using the standard recombinant DNA technique. Cells were seeded in 6-wells plate at $1 \times 10^{6} /$ well and allowed to attach for $24 \mathrm{~h}$ before transfection. Then 
transfection was processed by lipofectamine 2000(Invitrogen, USA) according to the manufacturer's protocol. Scramble primers were considered as scramble control. Cells treated with lipofectamine 2000 alone were set up as blank control. After incubation at $37^{\circ} \mathrm{C}$ and $5 \% \mathrm{CO}_{2}$ for $48 \mathrm{~h}$, cells were collected to testify the expression of Nrf2 by western Blot or for further study.

\section{Protein preparation}

Cytoplastic and nuclear protein were achieved using Nuclear and Cytoplasmic Protein Extraction Kit (Beyotime, Shanghai, China). Briefly, cells were processed in cytoplasmic protein extraction regent. Lysates were then incubated for $10 \mathrm{~min}$ on ice and centrifuged at $12000 \mathrm{~g}$ for $5 \mathrm{~min}$ at $4{ }^{\circ} \mathrm{C}$. The supernatant was harvested as the cytoplastic protein. Pellets containing crude nuclei resuspended in nuclear protein extraction regent were incubated and shaked for $2 \mathrm{~h}$ on ice. Samples were subsequently centrifuged at $12000 \mathrm{~g}$ for $10 \mathrm{~min} 4{ }^{\circ} \mathrm{C}$ to obtain supernatants containing nuclear protein. To obtain total protein lysate, cells or tissues were homogenized in RIPA buffer (Beyotime, Shanghai, China) with $1 \mathrm{mM}$ PMSF and centrifuged at $12000 \mathrm{~g}$ for $15 \mathrm{~min}$ at $4{ }^{\circ} \mathrm{C}$. Protein concentrations were estimated by Coomassie Plus Protein Assay Reagent (Pierce, USA).

\section{Western Blot Analysis}

Fifty micrograms of the protein extracts, such as cytoplastic and nuclear protein for Nrf2, total protein for heme oxygenase-1 (HO-1) and $\mathrm{NAD}(\mathrm{P}) \mathrm{H}$ :quinone oxidoreductase 1(NQO1) were heat denatured in loading buffer, separated by sodium dodecyl sulfate polyacrylamide gel electrophoresis, and electroblotted onto a nitrocellulose membrane. For immunoblotting, membranes were blocked with $5 \% \mathrm{BSA}$ for $2 \mathrm{~h}$ at room temperature, and the following antibodies were used: Nrf2 (sc-722, Santacruz, USA,68 kDa), H3(9715, CST, USA, 17 kDa), $\beta$-actin (AP0060, Bioworld, USA, 43 kDa), HO-1 (Ab13243, Abcam, USA, 32 kDa), NQ01 (Ab34173, Abcam, USA, 31 kDa), cleaved Caspase1(sc-22163, Santacruz, USA, 20 kDa), IL-1ß(NB600633,Novusbio, USA, 17 kDa), IL-18(sc-7954, Santacruz, USA, 18 kDa), NLRP2(sc-166584, Santacruz, USA, 121 kDa), p-IGF-IR(sc-81499, Santacruz, USA, 95 kDa), IGF-IR(sc-81167, Santacruz, USA, 95 kDa), AKT(9272, CST, USA, 60 kDa), p-AKT(4060, CST, USA, 60 kDa), m-TOR(2983, CST, USA, 289 kDa), pmTOR(5536, CST, USA, 289 kDa), MTNR1A(Ab203038,Abcam, USA,39 kDa). Each primary antibody was diluted appropriately in $5 \%$ BSA and then incubated overnight at $4{ }^{\circ} \mathrm{C}$. The blots were washed three times in the washing buffer and covered with suitable secondary antibody for $1 \mathrm{~h}$. Blots were incubated with enhanced chemiluminescence (ECL) detection system (Millipore,USA) and exposed by Tanon 5200 Chemiluminescence image analytical system (Tanon, Shanghai, China). $\beta$-actin was used as a housekeeping gene for total protein or cytoplasmic protein. $\mathrm{H} 3$ served as a housekeeping gene for nuclear protein.

\section{Transcription activity assay of Nrf2}

Nrf2 transcription activity was determined through TransAM Nrf2 kit (Active motif, CA, USA) according to manufacturer's instructions. Briefly, nuclear protein of different groups was collected after treatment and protein concentrations were estimated by Coomassie Plus Protein Assay Reagent (Pierce, USA). $10 \mu \mathrm{g}$ 
nuclear protein was used for TransAM Nrf2 assay. Absorbance were read on a spectrophotometer within 5 minutes at $450 \mathrm{~nm}$ with a reference wavelength of $655 \mathrm{~nm}$ (Bio-Rad Laboratories, USA).

\section{Flow cytometric analysis of pyroptosis}

Pyroptosis was identified as the presence of cleaved caspase-1 and PI double-positivity in FCM. Briefly, cells were washed twice and harvested in cold PBS after treatment. Following 15 min of incubation in antibody for cleaved Caspase 1 at room temperature, cells were washed twice with PBS, the secondary antibody labeled with FITC was incubated for $15 \mathrm{~min}$ in room temperature. After washing twice with PBS, cells were incubated with PI for another 15 min. Cleaved Caspase- 1 and PI double positive cells were detected by flow cytometry (Becton-Dickinson FACS Calibur, USA).

\section{ROS detection with DCFH-DA}

Intracellular ROS level was measured by fluorescent probe 6-carboxy-2', 7'-dichorodihydrofluorescein diacetate (DCFH-DA) according to manufacturer's instructions. Briefly, after 24 hours of treatment with different regent, cells were incubated with DCFH-DA in serum free medium for 10 minutes at $37^{\circ} \mathrm{C}$. Then cells were washed with PBS to remove the excess dye before fluorescent measurements that were carried out using the microplate reader fluoroscan ascent (Thermo scientific) with excitation and emission filters set at $485 \mathrm{~nm}$ and $538 \mathrm{~nm}$, respectively.

\section{Orthotopic tumor growth inhibition study}

Male BALB/c nude mice, weighing 18-20 g, were purchased from Shanghai Slac Laboratory Animal Co., Ltd. (Shanghai, China). All experimental procedures and animal care were approved by the Animal Care Committee of Jinling hospital. Athymic BALB/c nude mice (4-week-old, male) were used to establish orthotopic GBM model. Transfected U87MG-Luc cells (500,000 cells/mouse) were implanted stereotactically $1.0 \mathrm{~mm}$ anterior and $2.5 \mathrm{~mm}$ lateral to the bregma and at $3.5 \mathrm{~mm}$ depth from the skull surface. Seven days after transplantation, the mice was randomly divided into 5 groups: (1) $0.9 \%$ normal saline (NS), (2) DMSO(DMSO), (3) $25 \mathrm{mg} / \mathrm{kg}$ TMZ (TMZ), (4) $15 \mathrm{mg} / \mathrm{kg}$ MEL(MEL), (5) $25 \mathrm{mg} / \mathrm{kg}$ TMZ and $15 \mathrm{mg} / \mathrm{kg} \mathrm{MEL}(T M Z+M E L)(n=6)$. All drugs were given through intraperitoneal injection for consecutive 14 days. The administration time is 18 o'clock every day. The body weight of each mouse was measured. Caliper IVIS Spectrum was applied for in vivo bioluminescence imaging and luciferin was used as substrate for luciferase in 7 days and 21 days after transplantation. At $24 \mathrm{~h}$ after the final injection, all tumors were harvested for further analysis. And blood of nude mice was collected for alanine aminotransferase (ALT) and aspartate amino transferase (AST) analysis. The level of ALT and AST in nude mice serum was identified using commercially available kits (Jiancheng, Nanjing, China) according to manufacturer's instructions. In some experiments, propidiumiodide (PI) was injected i.p. into mice $1 \mathrm{~h}$ before tumor collection. Tumor after $\mathrm{PI}$ injection were performed for additional immunohistochemical analysis.

\section{Immunofluorescence analysis}


Immunofluorescence analysis was performed on frozen sections of xenograft tumor tissues after PI injection. Nonspecific antibody binding was blocked by incubating cells with $10 \%$ bovine serum albumin in PBS. Cells were incubated with cleaved Caspase1 primary antibody diluted 1:100 in 10\% BSA followed by fluorescently labeled secondary antibodies diluted 1:500 in PBST. Images were achieved with a laser scanning confocal microscope (FluoView, Olympus 1000, Center Valley, PA, USA). Secondary antibody alone was used as a negative control.

\section{Hematoxylin-eosin (HE) and Ki67 staining}

Brain tissues were fixed in $4 \%$ paraformaldehyde for $24 \mathrm{~h}$ at room temperature and then embedded in paraffin. Tissues were cut into 5 - $\mu \mathrm{m}$-thick sections. Then, sections were dewaxed with xylene and dehydrated with gradient ethanol. Slides were stained with HE. To investigate the tumor proliferation ability, we perform the Ki67 staining. Firstly, sections were blocked with $3 \% \mathrm{H}_{2} \mathrm{O}_{2}$ for 10 min at room temperature followed by incubation with primary antibody against Ki67(Ab15580,Abcam) at $4{ }^{\circ} \mathrm{C}$ overnight. Secondly, sections were washed in PBS followed by incubation with biotinylated secondary antibody (Dako, Denmark) at $25^{\circ} \mathrm{C}$ for $30 \mathrm{~min}$. Thirdly, sections were washed followed by incubation with streptavidin-peroxidase reagent (Dako) and 3, 3囚-diaminobenzidine (DAB; Sigma) mixture for 5 min. Finally, sections were counterstained and dehydrated for observation. Secondary antibody alone served as a negative control.

\section{Statistical analysis}

Data were expressed as mean \pm SD and evaluated by ANOVA and Turkey multiple comparison tests. $P \square$ 0.05 were considered to be significant. All analyses were performed by SPSS 18.0.

\section{Results}

\section{MEL receptor was enhanced in glioma tissue and cell lines}

It was reported that MEL receptor, MTNR1A, expressed in glioma. But its expression level was not yet clear. Herein, we detected the expression of MTNR1A in glioma tissue and cell lines. As showed in Fig. 1, expression of MTNR1A was elevated in glioma tissue as compared with it's in normal brain tissue(NB). Furthermore, MTNR1A was enhanced in high grade glioma(HGG) than it's in low grade glioma(LGG) (Fig. 1A,C). Such alteration was also demonstrated in glioma cell line as compared with it's in normal human astrocyte(HA)(Fig. 1B,D).

\section{MEL reduced glioma cell survival rate as co-treated with TMZ}

To evaluate the effect of MEL on glioma chemoresistance, cell survival rate of U251 and A172 treated with TMZ plus MEL in different concentration was identified through CCK-8 assay. Our results demonstrated that when treated with TMZ plus MEL, especially in TMZ plus $1 \mathrm{mM} \mathrm{MEL} \mathrm{group} \mathrm{at} 48 \mathrm{~h}$ 
time point, U251 (Fig. 2A)and A172(Fig. 2B) both showed decreased cell survival rate as compared with those in blank control group or TMZ group.

\section{MEL activated NLRP2 and enhanced pyroptosis induced by TMZ in glioma}

To reveal the mechanism under decreased cell survival rate in TMZ plus MEL(1 mM), we studied the pyroptosis rate after treatment. Pyroptosis was recognized as cleaved Caspase- 1 and PI double-positivity in FCM assay. Our results demonstrated that pyroptosis rate in TMZ + MEL group was much higher than those in TMZ or Control group (Fig. 3A,B). Also the ROS level in TMZ + MEL group was elevated as compared with those in TMZ or Control group (Fig. 3C).

Astrocytic NLRP2 inflammasome is an important component of the CNS inflammatory response[8]. Activation of NLRP2 leads to combination of NLRP2 inflammasome, which further induces cleavage of Caspase1 and mature of IL-1 $\beta$ and IL-18. Here we identified the expression of NLRP2 after treatment of $\mathrm{TMZ}+\mathrm{MEL}$. Western blot results indicated elevated expression of NLRP2 in TMZ + MEL group as compared with that of Control and TMZ group (Fig. 3D,E,F). Also enhanced expression of cleaved Caspase1, IL-1 $\beta$ and IL-18 was detected in TMZ plus MEL group (Fig. 3D,E,F).

\section{MEL reduced Nrf2 expression in glioma cells.}

$\mathrm{Nrf2}$ is an important protective transcript factor and play role in proliferation, apoptosis, autophage of glioma, which has been demonstrated in our previous studies. MEL can suppress the expression of ATPbinding cassette (ABC) transporter protein (ABCG2)[7], which has been found as downstream gene of Nrf2-ARE pathway[9]. So here we identified the effect of MEL on Nrf2 expression in glioma. Our results suggested that expression of Nrf2 in the cytoplasm and nucleus was reduced after MEL treatment in different concentration at $24 \mathrm{~h}(\mathrm{Fig} .4 \mathrm{~A}, \mathrm{~B}, \mathrm{C})$. Also we found decreased expression of Nrf2 after $1 \mathrm{mM} \mathrm{MEL}$ treatment in different time(Fig. 4D,E,F).

\section{MEL reversed elevation of Nrf2 expression and activity after TMZ stimulation through inhibition on IGF- IR/AKT/mTOR signal pathway.}

Chemotherapeutics can induce the elevation of Nrf2 expression in tumor, which further lead to chemoresistance. As reduced Nrf2 expression in glioma cells after treated with MEL in $1 \mathrm{mM}$ has been identified, we further studied whether MEL can reverse elevation of Nrf2 expression in glioma under TMZ stimulation. Western blot results revealed that after $24 \mathrm{~h}$ treatment TMZ induced elevation of Nrf2 expression in cytoplasm and nucleus along with downstream genes, such as NQ01 and HO1(Fig. 5A,B,C). And transcript activity of Nrf2 was also elevated after TMZ stimulation(Fig. 5D). But MEL significantly reversed elevation of Nrf2 expression in cytoplasm and nucleus stimulated by TMZ. The expression of NQ01 and HO-1 also decreased after TMZ plus MEL treatment(Fig. 5A,B,C). Also enhanced transcript activity of Nrf2 after TMZ stimulation significantly decreased under MEL treatment(Fig. 5D). 
It has been noted that Nrf2 can be regulated by IGF-IR/AKT/mTOR signal pathway[10]. Here we identified change of phosphorylation state of this signal pathway after treatment with TMZ plus MEL or not. Our results demonstrated that TMZ induced phosphorylation of IGF-IR/AKT/mTOR signal pathway, which could be reversed after MEL treatment (Fig. 5E,F,G).

\section{Enhanced Nrf2 expression reversed pyroptosis promotion effect of MEL}

Our results had demonstrated that MEL enhanced pyroptosis induced by TMZ in glioma through Nrf2 expression and transcription inhibition. To identify the role of Nrf2 in pyroptosis promotion effect of MEL, plasmid contained Nrf2 cDNA were transfected into U251 and A172. Western blot identified enhanced Nrf2 expression $48 \mathrm{~h}$ after transfection (Fig. 6A,B). After treatment with TMZ plus MEL, enhanced Nrf2 expression successfully reduced expression of NLRP2, cleaved caspase1, IL-1 $\beta$ and IL-18 (Fig. 6C,D,E), which suggested MEL may enhance pyroptosis through inhibition of Nrf2.

\section{MEL inhibited Nrf2 expression and promoted pyroptosis induced by TMZ in orthotopic GBM model.}

We confirmed that MEL promoted pyroptosis induced by TMZ in glioma through inhibition of Nrf2 expression and transcription activity. Here we further investigated such effect of MEL in orthotopic GBM model. After 7 days of intracranial orthotopic transplantation, the first in vivo fluorescence observation was carried out to confirm the tumor size. Then the mice were randomly divided into five groups. Nude mice received $25 \mathrm{mg} / \mathrm{kg} \mathrm{TMZ}(\mathrm{TMZ})$ or $15 \mathrm{mg} / \mathrm{kg} \mathrm{MEL}(\mathrm{MEL})$ or both (TMZ + MEL) i.p. for 14 days continuously. DMSO dissolved in normal saline same concentration served as control(DMSO). And only normal saline was injected as blank control(NS). Our results suggested that TMZ plus MEL successfully reduced tumor proliferation after 14 days treatment as compared with other groups(Fig. 7A, B). Total protein of tumor samples was harvested for western blot. The Nrf2 expression was enhanced after TMZ treatment. MEL treatment significantly decreased Nrf2 expression, which was still notable in TMZ + MEL group(Fig. 7E,G). MNTR1A, the receptor of MEL, was further investigated. The expression of MNTR1A was elevated after MEL treatment(Fig. 7E,F).

For mice received $\mathrm{PI}$ injection $1 \mathrm{~h}$ before tumor collection, immunofluorescence analysis for cleaved caspase1 and MNTR1A were performed. Enhanced co-expression of cleaved caspase1 (green) and PI (red) in cytoplasm was observed in TMZ + MEL group(Fig. 7I). Also enhanced MNTR1A was identified in TMZ + MEL group(Fig. 7I). HE stain showed significantly decreased tumor size in TMZ + MEL group as compared with those in all the other groups(Fig. 7J). Also Ki67 stain demonstrated diminished proliferation in TMZ + MEL group(Fig. 7J). To identify the possibly liver damage after treatment of TMZ + MEL, here we collected mice blood for ALT and AST analysis while tumor collection. The level of ALT and AST showed slight elevation as compared with those in other groups. But no significant difference was discovered(Fig. 7D, H). The body weight of mice showed a slight decline in all groups (Fig. 7C).

\section{Discussion}


Nrf2 is a traditional antioxidant transcript factor, which plays important cytoprotective effect against many kinds of stress. For recent years, it has been discovered that Nrf2 also protects tumor cells against chemotherapeutic[11, 12]. Inhibition of Nrf2 expression and activity through brusatol, a unique inhibitor of the Nrf2, successfully enhanced chemosensitivity in a broad spectrum of cancer cells and A549 xenografts[12]. Our previous studies also identify that Nrf2 participates in proliferation, migration, apoptosis and chemoresistance of glioma[13-17]. Here we provided MEL as a new Nrf2 inhibitor, which has been approved for clinical use by FDA. Previous literature reports that MEL can activate the Nrf2-ARE pathway through multiple pathways and further attenuate oxidative stress[18]. However, in our study, it was found that MEL may reduce the phosphorylation level of the IGF-IR/AKT/mTOR pathway and reversing the high level of Nrf2 expression in glioma, which would ameliorate the chemosensitivity. The concentration of MEL as a Nrf2 agonist is almost in $\mathrm{nM}$ level[19], which is in the physiological concentration. Our results confirmed that MEL in $1 \mathrm{mM}$ could remarkably reduce basic Nrf2 expression in cytoplasm and nucleus in U251 and A172. TMZ treatment activated Nrf2-ARE pathway and induced elevation of downstream genes, such as HO-1 and NQ01. But enhanced expression of Nrf2 and its downstream genes after TMZ stimulation can also be inhibited by MEL. Transcript activity of Nrf2 elevated after TMZ stimulation also diminished after MEL treatment. These results confirm that TMZinduced elevation of Nrf2 can be reversed by MEL, thereby increasing chemosensitivity.

Melatonin, an indolamine produced and secreted predominantly by the pineal gland, mediates a variety of physiological functions and possesses antioxidant and antitumor properties. Physiologically, melatonin is secreted at low (nanomolar) concentrations, and has been shown to be an effective antioxidant, free radical scavenger, and regulator of antioxidant genes. Previous studies have shown that MEL at high (millimolar) concentrations(in or more than $1 \mathrm{mM}$ ) can dramatically inhibit tumor proliferation and migration[20, 21]. Here we used MEL in pharmacological concentration (1 mM), which has been recognized as antitumor concentration[7,22]. Our results indicated that MEL in $1 \mathrm{mM}$ can enhance the antitumor effect of TMZ in glioma. After treatment of TMZ plus MEL, glioma cell showed more pyroptosis as compared with those only treated with TMZ. Also diminished orthotopic GBM model was identified after treated with TMZ plus MEL. Furthermore, our results also demonstrated the MEL receptor MTNR1A, also named as MT1, expressed in glioma tissue and cell lines. The level of MTNR1A was elevated in HGG than it's in LGG. Recently, it has been reported that MTNR1A was abundant in PTEN wt glioma than it's in PTEN mut glioma[23]. And elevated MTNR1A in PTEN wt glioma was associated with better anti-tumor effect from MEL, which indicated that enhanced MTNR1A may promote the chemosensitizer effect of MEL.

Pyroptosis is a process of inflammatory cell death initiated by inflammasome[24]. Different from apoptosis, the process of pyroptosis is induced by NLRP family or AIM2, which induces ASC-Caspase 1 to form inflammasome, promotes Caspase 1 cleavage, and further induces the release of IL-1 $\beta$ and IL-18. Previous studies have shown that Nrf2 is involved in the regulation of cell death pyroptosis, and its role involves the regulation of ROS production by Nrf2[25, 26]. Nrf2 can reduce intracellular ROS levels, thereby reducing the occurrence of pyroptosis. Our results demonstrated that MEL at mM levels can 
inhibit the expression of Nrf2 in glioma. So it is possible that MEL may promote the occurrence of pyroptosis by inhibiting Nrf2 in glioma.

It has been demonstrated that miR-214 regulates the pyroptosis process in glioma[27]. However, there is no report on whether TMZ, a commonly used chemotherapy drug, can regulate pyroptosis in the glioma. For the first time, our results reported that TMZ can induce the pyroptosis in glioma, and this process may be associated with increased expression of NLRP2. The formation of pyroptosis is mostly induced by NLRP3, but NLRP3 is expressed less in astrocytes, while NLRP2 is thought to be highly expressed in astrocytes[24]. Our results also revealed that the process of pyroptosis in glioma, which is associated with activation of NLRP2 and increased ROS level.

\section{Conclusions}

In summary, millimolar concentration of MEL can inhibit the expression of Nrf2 in glioma, and the mechanism may involve the IGF-IR/AKT/mTOR pathway dephosphorylation process. By inhibiting the expression of Nrf2, MEL can further promote TMZ-induced glioma pyroptosis, accompanied by increased expression of cleaved caspase $1, \mathrm{IL}-1 \beta$ and IL-18. Therefore, as an approved drug by the FDA, MEL has the potential to become a sensitizer for chemotherapy and improve the prognosis of patients with glioma.

\section{Abbreviations}

GBM

glioblastoma; MEL:melatonin; TMZ:temozolomide; GICs:glioblastoma-initiating cells; HO-1:heme oxygenase-1; NQ01:NAD(P)H:quinone oxidoreductase 1; ALT:alanine aminotransferase; AST:aspartate amino transferase; PI:propidiumiodide; HE:Hematoxylin-eosin; HGG:high grade glioma; LGG:low grade glioma; HA:human astrocyte; ABC:ATP-binding cassette;

\section{Declarations}

\section{Availability of data and materials}

All data generated or analyzed during the current study are included in this published article.

\section{Ethics approval and consent to participate}

The specimens of patients with glioma were conducted with permission from the Institutional Research Ethics Committee of Jinling hospital. All animal studies were conducted with the approval of the Jinling hospital Institutional Animal Care and Use Committee.

\section{Consent for publication}

Not applicable. 


\section{Competing interests}

The authors declare that they have no competing interests.

\section{Funding}

Supported by the National Natural Science Foundation of China (Grant No. 81402072, Grant No.81672503), Young Medical Talents in Jiangsu Provinc(QNRC2016898). The funders had no role in study design, data collection and analysis, decision to publish, or preparation of the manuscript.

\section{Author's contribution}

HD.W and LB. C were involved in study design, data interpretation and manuscript editing; H.P and Q. W performed the majority of the laboratory work and contributed to the analysis of data and writing of the manuscript; KJ. S and Q. W contributed to the analysis of data; $\mathrm{Y}$. $\mathrm{J}$ were responsible for the cell culture and part of laboratory work; WH. N was involved in manuscript editing. All authors declared no conflict of interest.

\section{Acknowledgements}

We are very grateful to the patients and mice for their contributions and sacrifices to this study.

\section{References}

\section{Acknowledgements}

We are very grateful to the patients and mice for their contributions andsacrifices to this study.

\section{Funding}

Supported by the National Natural Science Foundation of China (Grant No. 81402072,Grant No.81672503), Young Medical Talents in Jiangsu Provinc(QNRC2016898). The funders had no role instudy design, data collection and analysis, decision to publish, orpreparation of the manuscript.

\section{Author'scontribution}

HD.W and LB. C were involved in study design, data interpretationand manuscript editing; H.P and Q. W performed the majorityof the laboratory work and contributed to the analysis ofdata and writing of the manuscript; KJ. Sand Q. Wcontributed to the analysis of data; Y. Jwereresponsible for the cell culture and part of laboratory work; WH. N was involved in manuscript editing. All authorsdeclared no conflict of interest. 
Availability of data and materials

All data generated or analyzed during the current study are included in thispublished article.

Ethics approval and consent to participate

The specimens of patients with glioma were conducted with permission fromthe Institutional Research Ethics Committee of Jinling hospital. All animal studies were conducted with theapproval of the Jinling hospital Institutional Animal Care and UseCommittee.

\section{Consent for publication}

Not applicable.

\section{Competing interests}

The authors declare that they have no competing interests.

\section{Figures}

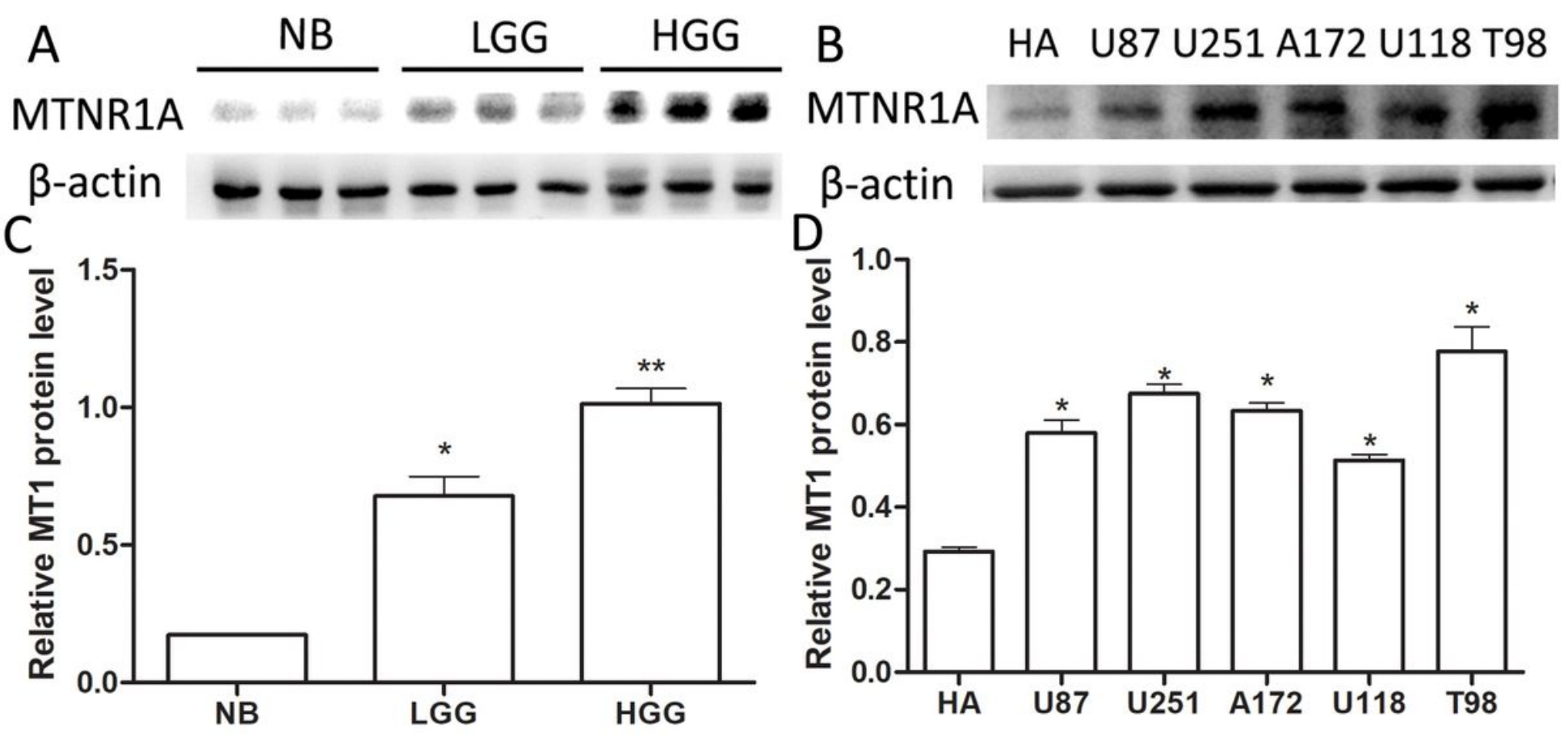

\section{Figure 1}

Increased expression of MTNR1A in glioma cells and tissues. Enhance expression of melatonin receptor, MTNR1A, in LGG and HGG as compared with it's in normal brain $(A, B)$. Expression of MTNR1A was also elevated in different glioma cell lines as compared with it's in normal human astrocyte $(A, C) .{ }^{*} p<0.05$ as compared with NB or $\mathrm{HA} ;{ }^{\star \star} \mathrm{p}<0.05$ as compared with LGG. 

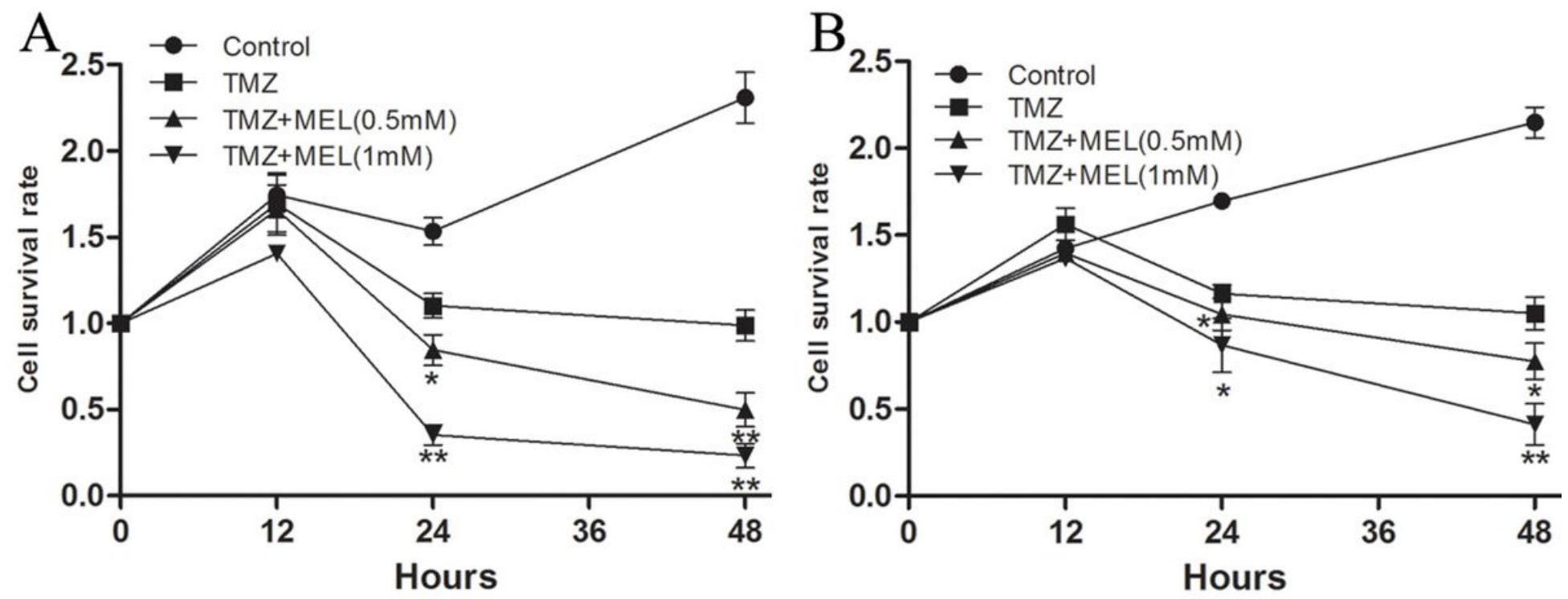

Figure 2

Melatonin promotes the killing effect of TMZ on glioma. TMZ in combination with $0.5 \mathrm{mM}$ or $1 \mathrm{mM} \mathrm{MEL}$ simultaneously acts on U251 (A) or A172 (B), which results in more cell death at $48 \mathrm{~h}$ compared to TMZ alone. * $\mathrm{p}<0.05$ as compared with control; ${ }^{* *} \mathrm{p}<0.05$ as compared with $\mathrm{TMZ}$ alone
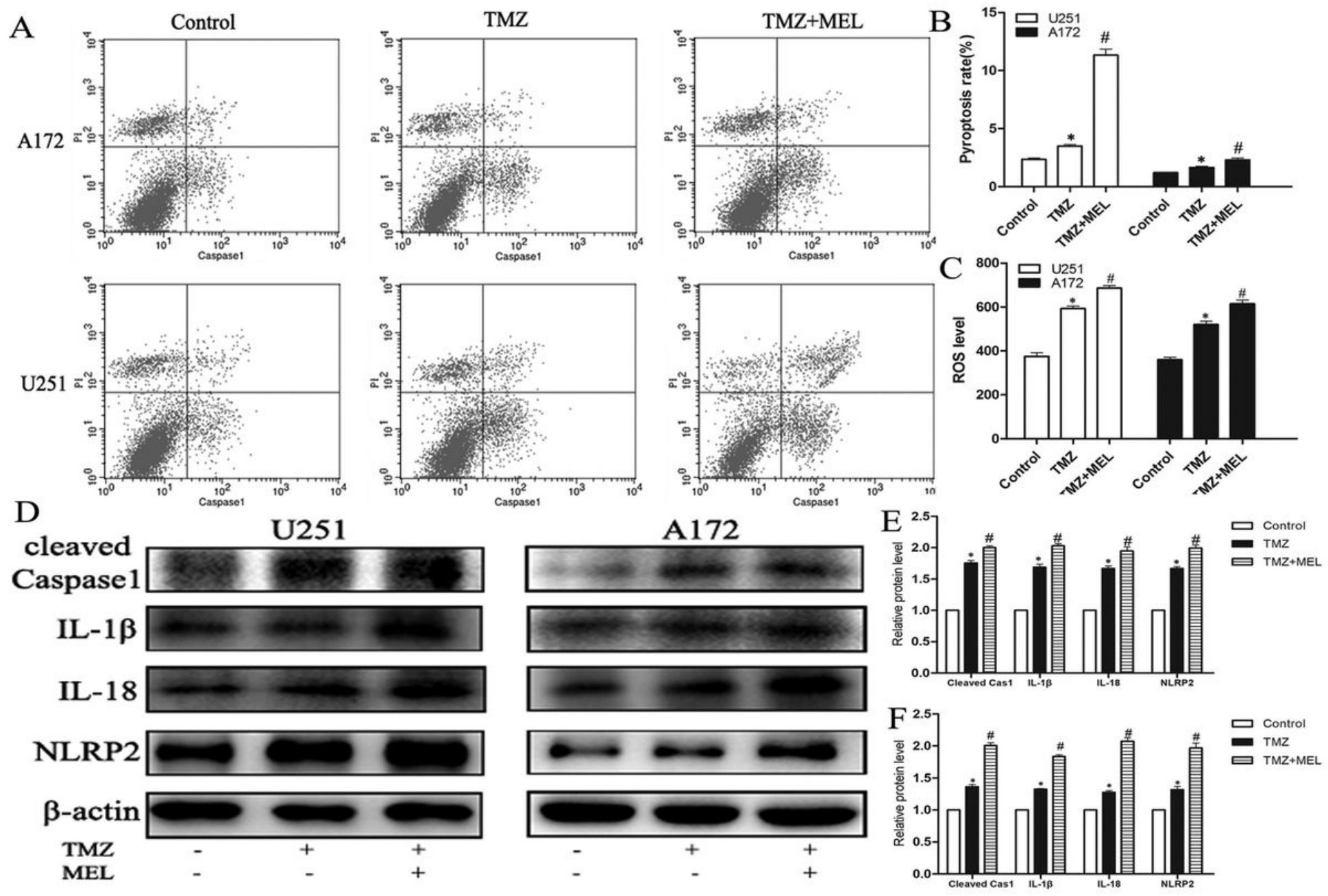


\section{Figure 3}

MEL promotes TMZ-induced glioma pyroptosis. Flow cytometry results showed that PI plus cleaved Caspase1-positive cells were significantly increased (A. B) compared with those treated with TMZ alone, and intracellular ROS levels were also significantly increased(C). After MEL combined treatment with TMZ, the NLRP2, cleaved Cas1, IL-1 1 , and IL-18 were significantly increased compared with those in TMZ alone group(D,E for $\mathrm{U} 251$ and $F$ for $A 172)$. * $p<0.05$ as compared with control; \#p<0.05 as compared with TMZ alone
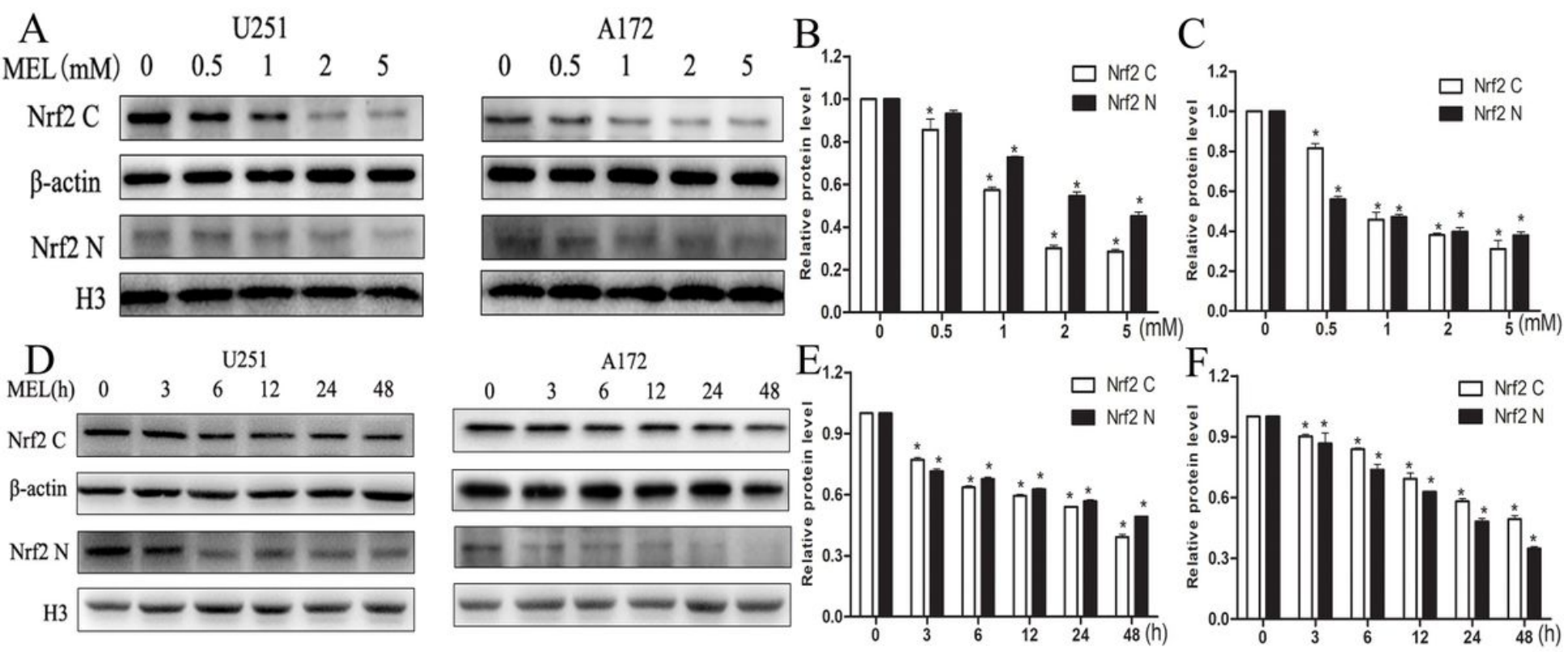

\section{Figure 4}

MEL inhibits Nrf2 expression in gliomas. Nrf2 protein levels were detected $24 \mathrm{~h}$ after different concentrations of MEL treatment(A). It was found that Nrf2 levels decreased with increased MEL concentration(B for U251 and C for A172). In addition, after $1 \mathrm{mM}$ MEL treatment, Nrf2 protein levels were observed at different time points(D). It was found that Nrf2 levels gradually decreased with time(E for $\mathrm{U} 251$ and $\mathrm{F}$ for $\mathrm{A} 172) .{ }^{*} \mathrm{p}<0.05$ as compared with control. 


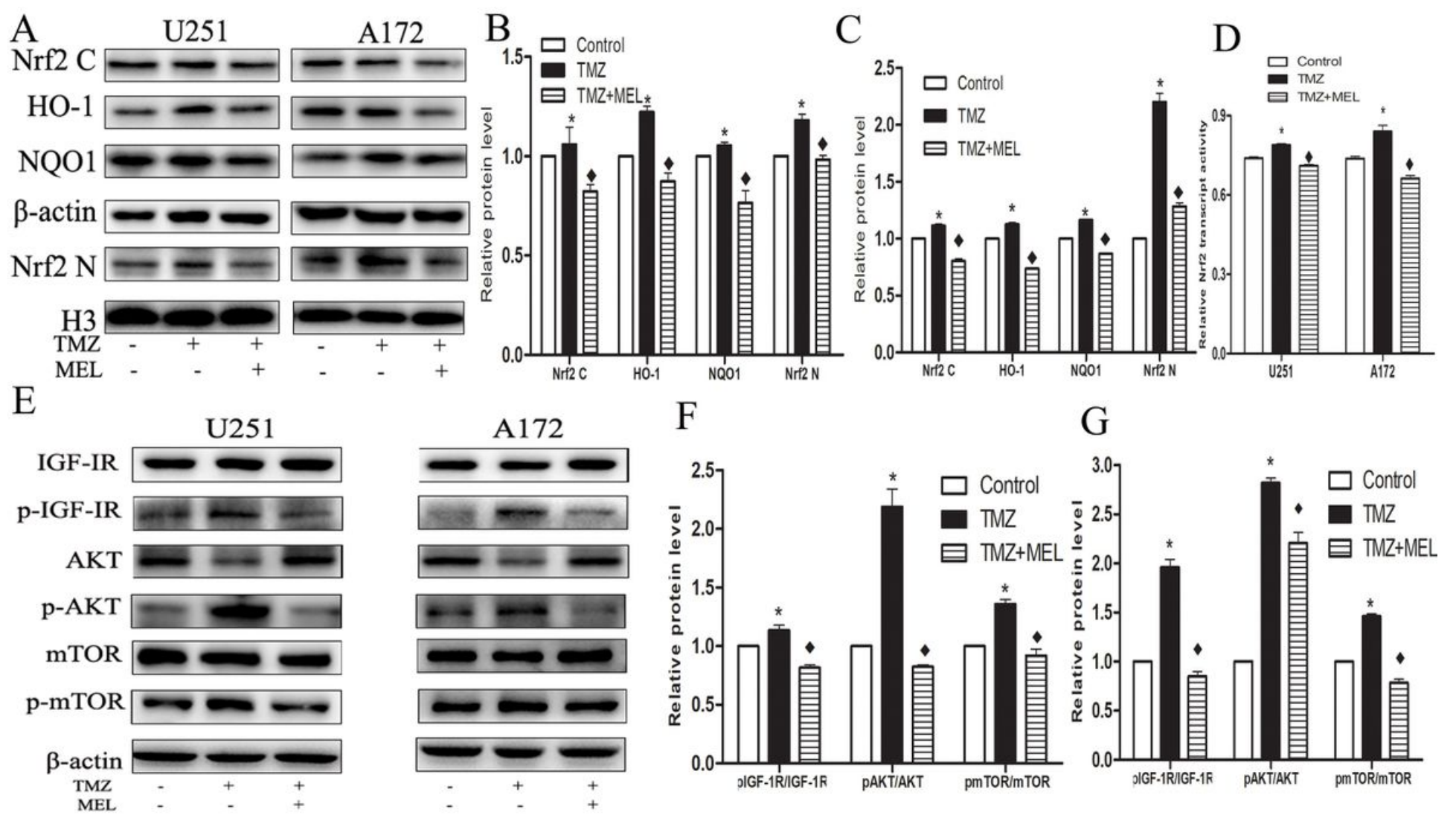

\section{Figure 5}

MEL down-regulates Nrf2 expression by inhibiting phosphorylation of the IGF-1R/AKT/mTOR pathway. The combination of MEL and TMZ can reduce the up-regulation of TMZ-induced elevation of Nrf2 and its downstream HO-1 and NQ01 expression levels(A, B for U251 and C for A172). At the same time, TMZinduced up-regulation of Nrf2 transcriptional activity was also decreased(D). The realization of this role of MEL may be related to the inhibition of phosphorylation of the IGF-1R/AKT/mTOR pathway. $(E, F$ for U251 and $G$ for A172) . * $p<0.05$ as compared with control; $\otimes p<0.05$ as compared with $T M Z$ alone 


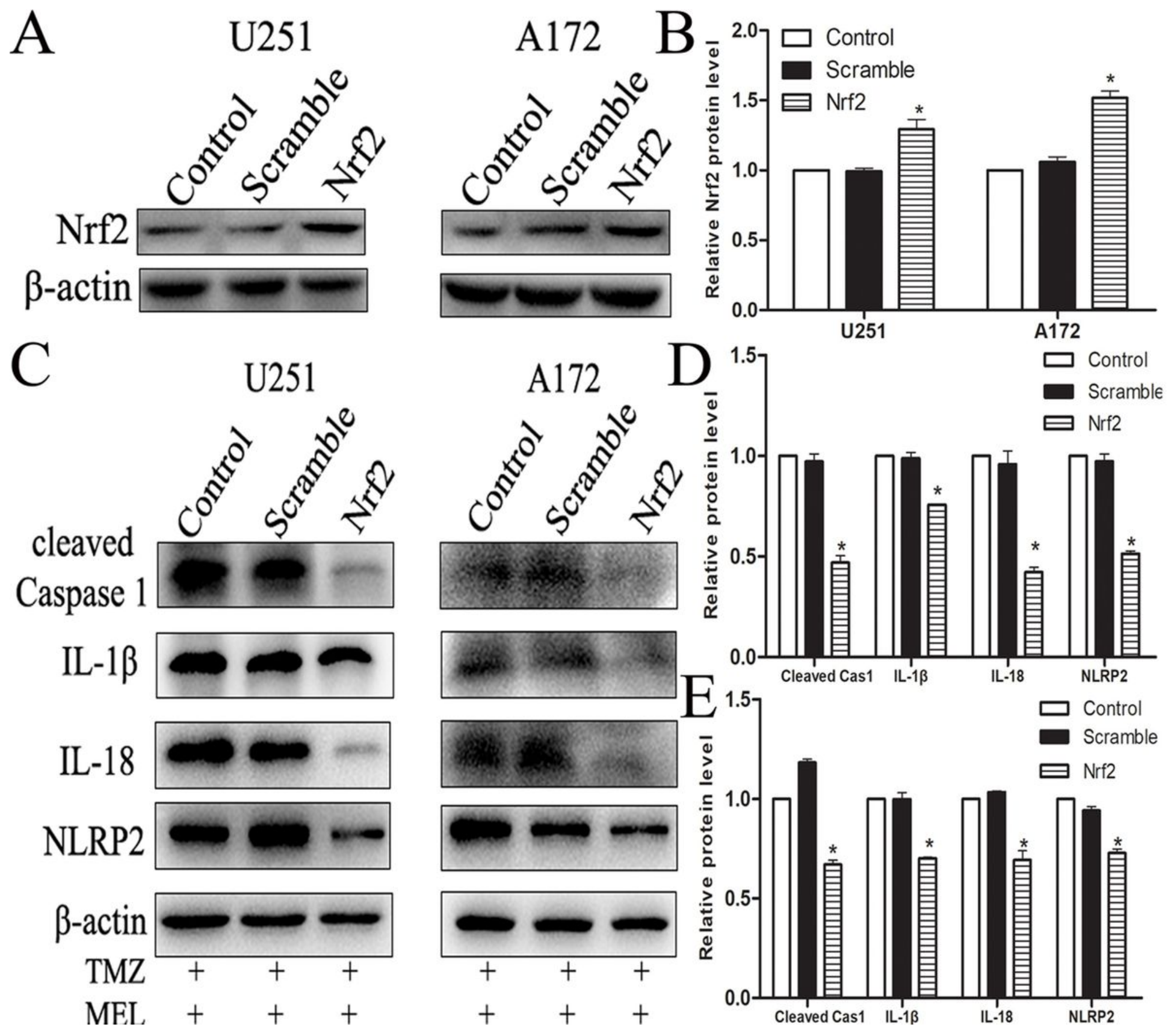

Figure 6

Up-regulation of Nrf2 expression reverses the pro-pyroptosis effect of MEL. The expression level of Nrf2 in $\mathrm{U} 251$ and $\mathrm{A} 172$ was up-regulated by plasmid transfection(A,B). After up-regulation of Nrf2 expression, the combination of TMZ and MEL treatment induced pyroptosis-related proteins were significantly reduced as compared with those in TMZ treatment alone(C, D for U251 and E for A172). ${ }^{*} p<0.05$ as compared with TMZ. 
A NS DMSO TMZ

MEL TMZ+MEL

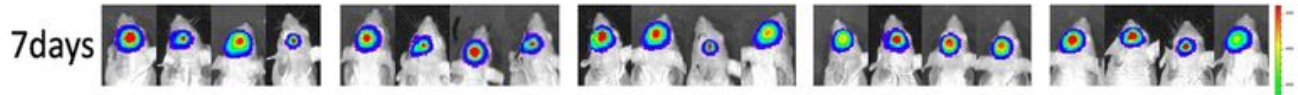

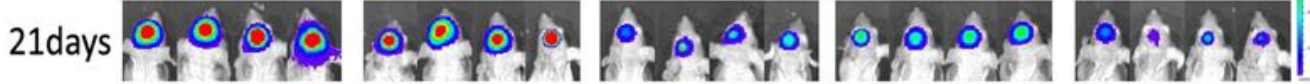
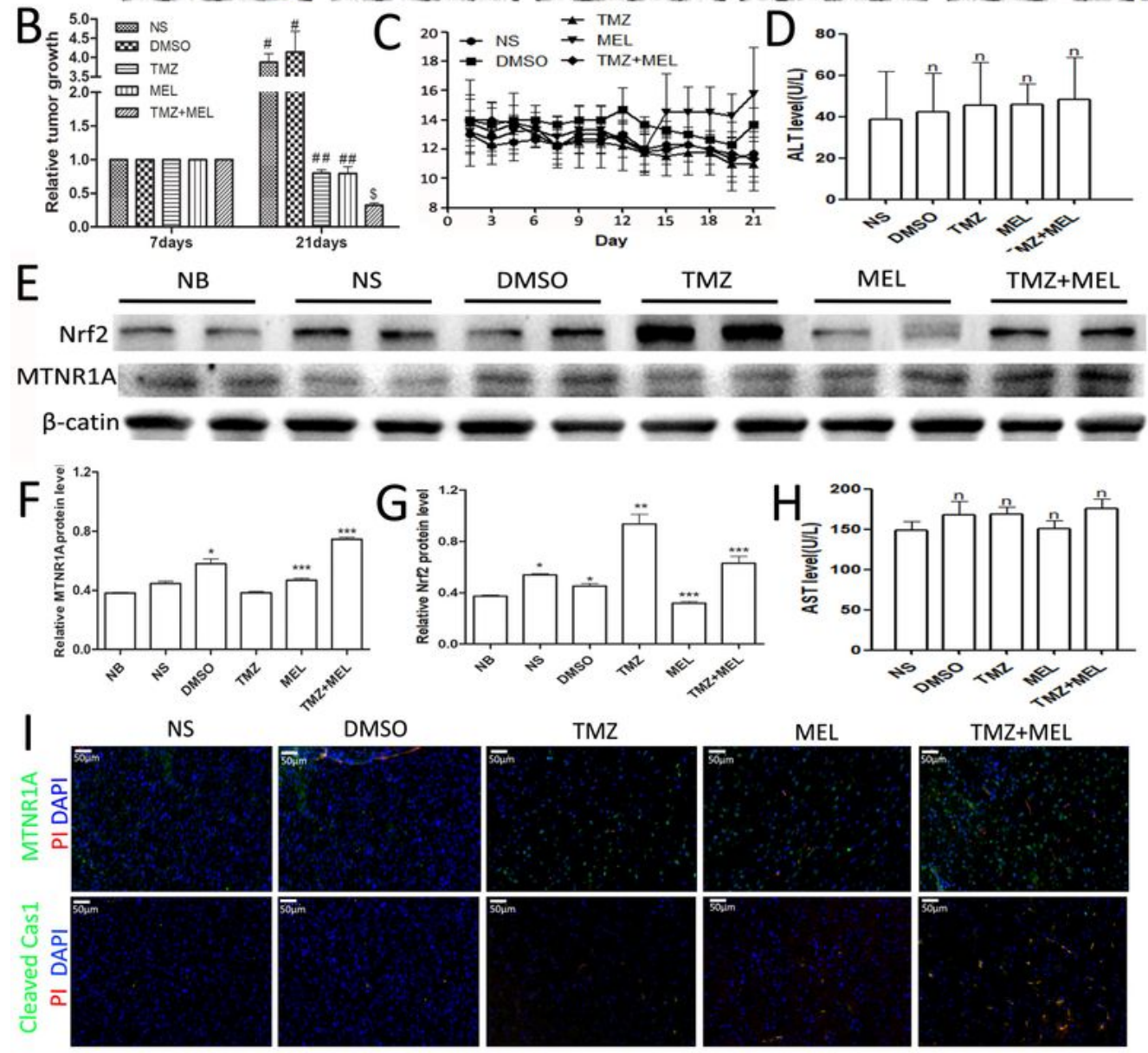

DMSO
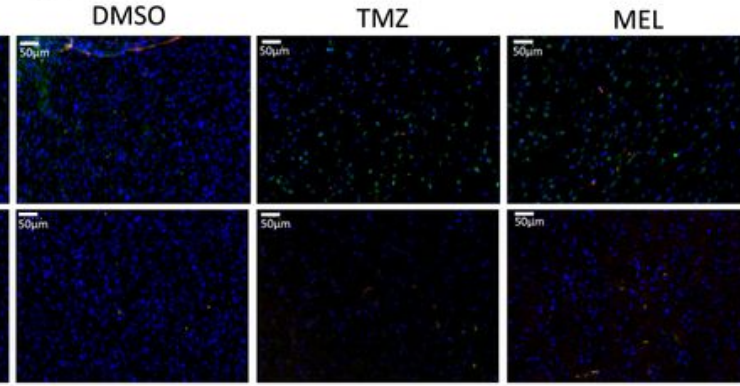

$\mathrm{TMZ}+\mathrm{MEL}$

J

DMSO

TMZ

MEL
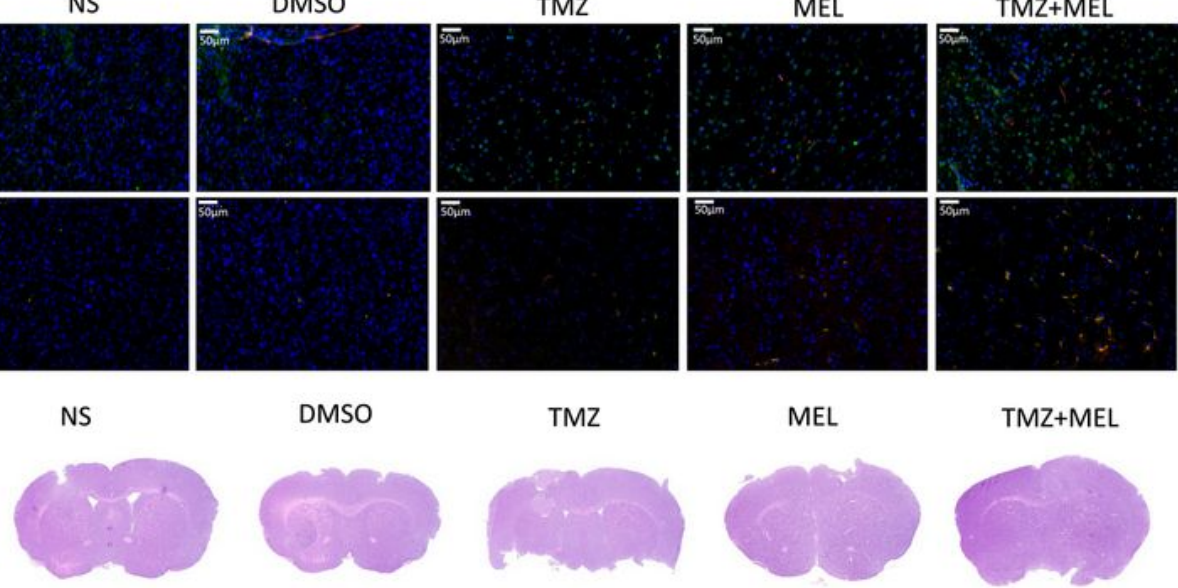

TMZ+MEL
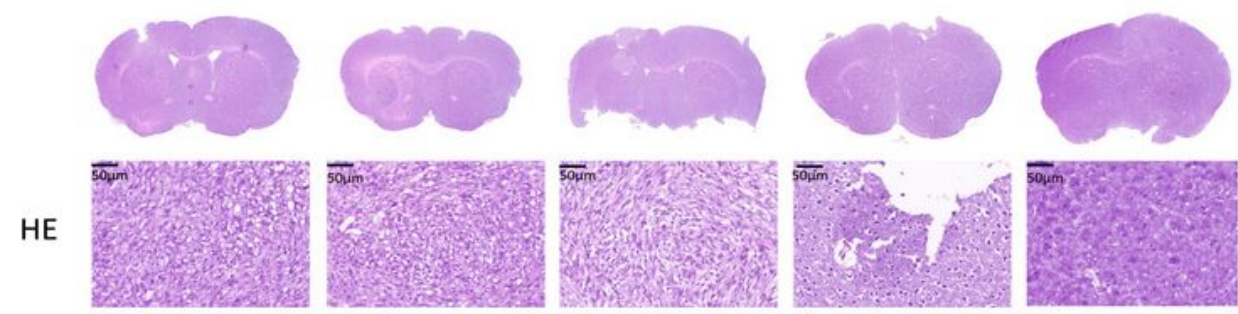

Ki-67
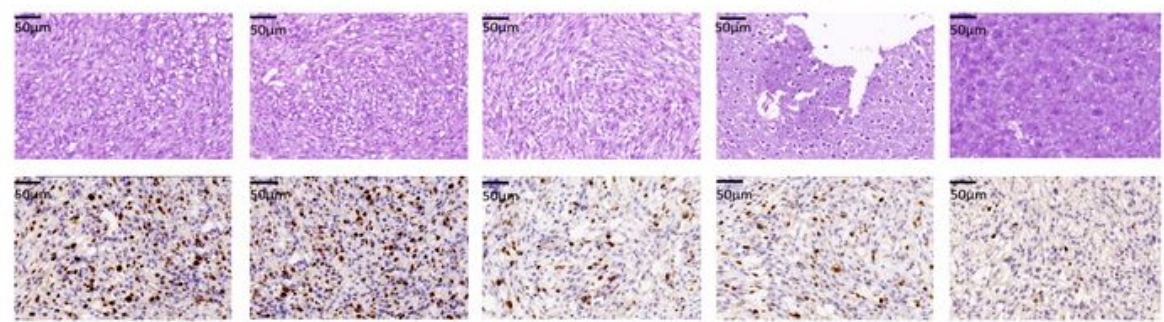

50, 3

$50 \mathrm{pin}$
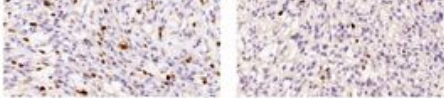

\section{Figure 7}

In vivo experiments confirmed that MEL promote TMZ-induced glioma pyroptosis. The combined effect of $25 \mathrm{mg} / \mathrm{kg} \mathrm{TMZ}$ and $15 \mathrm{mg} / \mathrm{kg}$ MEL for 14 days showed a significant reduction in the volume of the transplanted tumor compared with the effect of TMZ or MEL alone(A,B). The expression level of Nrf2 and MT1 was detected. Compared with TMZ group, MEL significantly decreased Nrf2 expression and reversed the increase of Nrf2 induced by TMZ $(E, G)$. And MTNR1A expression in TMZ+MEL group was enhanced 
as compared with TMZ or MEL alone(E,F). There were no significant changes in ALT, AST and body weight in mice $(\mathrm{C}, \mathrm{D}, \mathrm{H})$. Fluorescent staining showed that $\mathrm{PI}$ and cleaved Cas1 double positive cells (pyroptosis cells) in the tumor increased significantly in the TMZ combined MEL group as compared with TMZ or MEL treatment alone(I). Coronal section of rat brain and HE stain showed significantly reduced tumor in TMZ+MEL group $(J)$. Also Ki67 stain showed decreased proliferation index in TMZ+MEL group as compared with TMZ or MEL alone $(J)$. ${ }^{\star} p<0.05$ as compared with NB. ${ }^{* \star} p<0.05$ as compared with NS or DMSO. ${ }^{\star \star \star} p<0.05$ as compared with TMZ. \# $p<0.05$ as compared with itself's group in 7day. \#\# $p<0.05$ as compared with NS or DMSO in 21 day. $\$ p<0.05$ as compared with other groups in 21day. NB: normal brain around the tumor of the NS group. 